\title{
Risk Factors for Prematurity among Neonates from HIV Positive Mothers in Cameroon
}

\author{
Taguebue $\mathrm{J}^{1,2}$, Monebenimp $\mathrm{F}^{2}$, Zingg $\mathrm{W}^{1}$, Mve Koh $\mathrm{V}^{3}$, Atchoumi $\mathrm{A}^{3}$, Gervaix $\mathrm{A}^{1}$, Tetanye $\mathrm{E}^{2}$ \\ ${ }^{1}$ Department of Pediatrics and Adolescent Medicine, University Hospitals of Geneva, Geneva, Switzerland; ${ }^{2}$ Department of Pediat- \\ rics, University of Yaoundé Hospital, Yaoundé, Cameroon; ${ }^{3}$ Department of Gynecology and Obstetrics, University of Yaoundé Hos- \\ pital, Yaoundé Cameroon. \\ Email: walter.zingg@hcuge.ch
}

Received March $11^{\text {th }}, 2011$; revised March $15^{\text {th }}$, 2011; accepted March $18^{\text {th }}$.

\begin{abstract}
Objective: Human immunodeficiency virus (HIV) infection is the most important chronic health condition among women in childbearing age in sub-Saharan Africa. There is sparse data about socio-economic factors in the context with adverse outcome in pregnancy. The objective of the study was to identify such factors for prematurity, low birth weight (LBW) and intrauterine growth retardation (IUGR) in infants born to HIV-positive women in Cameroon. Methods: The study was done in Yaoundé between December 2001 and November 2007. Neonates born to HIV-positive mothers were monitored and clinical data as well as socio-economic factors were tested for association with prematurity, LBW and IUGR. Findings: In total, 264 neonates were analyzed. More prematurity occurred when mothers attended less than 4 prenatal care visits (OR [95\% CI]: 2.7 [1.21 - 6.05]; $p=0.015)$. HIV-classification $>1$ resulted in more IUGR (OR [95\% CI]): 3.15 [1.4 - 7.4]; $p=0.01)$ and LBW (2.20 [1.1 - 4.6]; $p=0.03)$. Single women were more likely to attend 4 prenatal care visits or more (OR [CI95\%]: 2.4 [1.6 - 3.4]; $p<0.001)$; higher education resulted in better compliance with anaemia prophylaxis (OR [CI95\%]: 3.0 [1.5 - 5.8]; $p=0.002)$ and antimalaria prophylaxis $(O R$ [CI95\%]: 2.1 [1.4 - 3.3]; $p<0.001)$; and was associated with early HIV diagnosis $(p=0.003)$. Conclusion: Prenatal care improves outcome in pregnancy of HIV-positive women. Single mothers compared to women in family settings are more likely to attend prenatal care visits, and HIV was diagnosed earlier in pregnancy in this population. More effort should be put to address women in family settings to early diagnose HIV and to increase compliance with prenatal care.
\end{abstract}

Keywords: Neonates, HIV, Pregnancy, Prematurity, Low Birth Weight, Intrauterine Growth Retardation, Cameroon, Africa, Education

\section{Introduction}

Low birth weight (LBW), prematurity and intrauterine growth (IUGR) is associated with considerable morbidity and mortality in the neonatal period as well as in the first year and adult life [1-5]. LBW often is due to IUGR, prematurity or a combination of both. However, other factors such as a lower socio economic status, a singleparent family, extreme maternal age at delivery $(<20$ or $>34$ years), a lower education level, short intervals between pregnancies, multiple pregnancy, tobacco and/or alcohol use during pregnancy, low weight gain during pregnancy, and chronic illness of the mother have been associated with LBW as well [6-10].

Human immunodeficiency virus (HIV) infection with or without acquired immunodeficiency syndrome (AIDS) is the most important chronic health condition among women in childbearing age in sub-Saharan Africa with an estimated 12.2 millions women affected. The overall HIV prevalence in Cameroun is $5.5 \%$. The prevalence among women in childbearing age shows geographical distribution between $5.7 \%$ up to more than $12 \%$ [11]. Studies from central Africa found that HIV-positive mothers are especially at risk for preterm childbirth or giving birth to a child with IUGR or LBW [12-15]. There is a direct correlation between vertical HIV transmission, LBW and a high mortality [16-19].

Before 2008, ART was not available free of charge for the HIV-treatment during pregnancy in Cameroon. However, standardized HIV prophylaxis with nevirapine and zidovudine was offered at delivery. All newborns were treated with nevirapine single dose alone (before 
2003) or in combination with zidovudine for 4 weeks (from 2003) [20]. With the introduction of such precaution measures for vertical transmission more and more women were offered an HIV-test during pregnancy. A steady increase of HIV-testing revealed to what extent of offspring was at risk for HIV and related health conditions in Cameroon. The aim of this study was 1) to identify LBW, IUGR and prematurity of children born to HIV-positive mothers; and 2) to identify modifiable risk factors associated with LBW, IUGR and prematurity of infants born to HIV-positive women.

\section{Methods}

Setting: This prospective cohort study was done at the University of Yaoundé Teaching Hospital (UYT), one of four university hospitals in Yaoundé. The department of gynaecology and obstetrics at the UYT has an estimated 2250 deliveries per year.

Inclusion criteria: all neonates born to HIV positive mothers were prospectively included in the study between December 2001 and November 2007 if: 1) they were born at UYT; 2) has an HIV-positive mother; who 3) attended at least one prenatal care visit at UYT. Only cases with at least $75 \%$ of data completed as required by the case related form (CRF) were included in the final analysis.

Variables: Clinical data of neonates and mothers included in the study was obtained from patient charts. All mothers were checked clinically (fatigue, pallor) for anaemia on the occasion of prenatal care visits and at delivery. Haemoglobin levels were checked only if clinical signs for anaemia were present. Syphilis was routinely screened with a VDRL test at least once at the visit during pregnancy.

Definitions: LBW was defined as a birth weight $<2500$ g. Prematurity was defined as a gestational age below 37 weeks. IUGR was defined as a birth weight below the $10^{\text {th }}$ percentile of gestational age based on the UK National Healthcare Care intrauterine growth curve. The weight-to-length ratio (WLR) was calculated using the Rohrer's ponderal index $\left(100^{*}\right.$ weight/length ${ }^{3}$ where weight is indicated in grams and length in centimetres) [21]. A mother was considered HIV-positive when at least two different HIV antibody tests (either for HIV-1 or for HIV-2) were positive or if one positive antibody test was confirmed by a western-blot. Time of HIV-diagnosis was stratified into four groups: 1) before pregnancy; 2) during the first trimester; 3) during the second trimester; and 4) during the third trimester of pregnancy. Clinical stage of HIV disease was classified using the WHO clinical classification [22]. Antiretroviral therapy (ART) was defined as any combination of at least three antiretroviral drugs regardless of specific drug combina- tions or whether the substances were active or not. When available, HIV viral load, total lymphocyte cell counts, CD4 cell counts, and CD8 cell counts were recorded. The number of prenatal care visits was stratified into trimesters and a maximum of two visits per trimester was counted. Socioeconomic parameters such as matrimonial status, regular income and source of income of the mother were obtained from the patient files. Compliance with malaria prophylaxis was obtained from the patient charts and defined as sleeping under a mosquito net and/or taking an intermittent treatment of $1500 \mathrm{mg}$ sulfadoxine-pyrimethamine every 8-12 weeks beginning at 16 weeks of pregnancy. Malaria was suspected if the mother had a fever $\left(>38^{\circ} \mathrm{C}\right)$ during the pregnancy or at the time of delivery and confirmed if Plasmodium falciparum was detected in the blood or when symptoms resolved after anti-malaria treatment in the absence of other antimicrobial agents. Anaemia prophylaxis was defined as starting intake of iron and folic- acid daily in the third trimester or before. Anaemia was defined as haemoglobin of below $11 \mathrm{~g} / \mathrm{l}$. Syphilis was considered positive if a positive VDRL test was confirmed by a TPHA test. The diagnosis of eclampsia or preeclampsia was done on clinical grounds.

Statistical Analysis: Sample size calculation was done using US-data and data from Rwanda as specific data on LBW in Cameroun are not available[19,23]. Categorical variables were compared using the $\mathrm{x}^{2}$ test; continuous variables were summarized as means or medians and compared using the Wilcoxon rank sum test. We used logistic regression to investigate the association between potential risk factors and IUGR as defined as birth weight $<10^{\text {th }}$ percentile. We first investigated all potential risk factors in univariate analysis. Variables associated with an increased risk for IURG with a p-value $<0.2$ were analyzed in multivariate analysis. A p-value $<0.05$ was considered statistically significant and only variables meeting this criteria were kept in the final model. All statistical analyses were conducted using Stata 10.0 (Stata Corporation, College Station, Texas; USA).

\section{Results}

Enrolment: Among a total of 15379 children born at UYT during the study period, 496 children were born to HIV-1-positive mothers (Figure 1): 119 neonates were excluded because their mothers did not have prenatal care at UYT and therefore information about pregnancy was not obtainable; 113 neonates were excluded from final analysis because documents were less than $75 \%$ complete. A total of 264 neonates were included in the final analysis (Figure 1).

Patient Characteristics: A total of 264 neonates with a mean gestational age of 38.7 (SD: \pm 2.5 weeks) were 


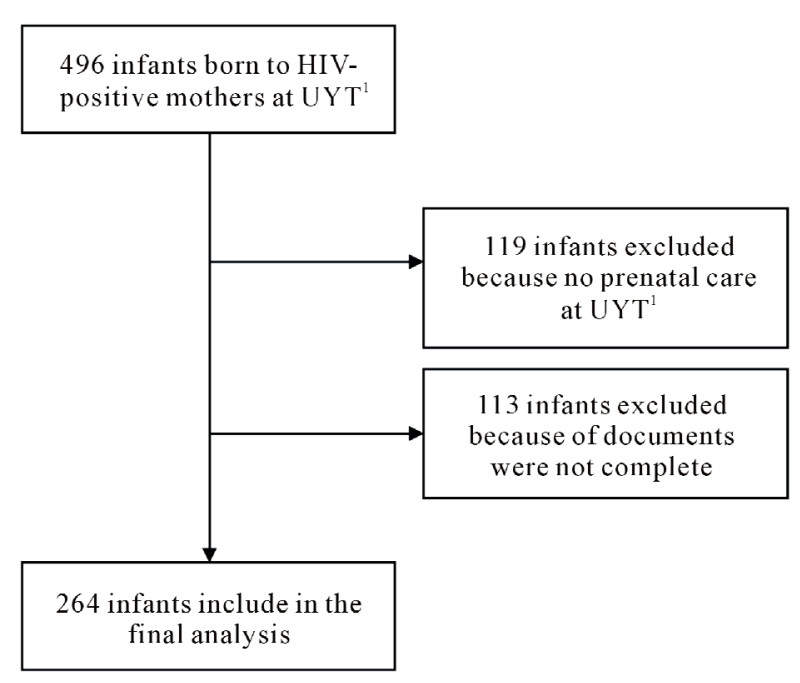

Figure 1: Enrolment of neonates from HIV infected mothers for the study on risk factors of low birth weight (LBW) and intrauterine growth retardation (IUGR), University of Yaoundé Teaching Hospital, Cameroon (December2001November 2007). ( ${ }^{1}$ UYT: University of Yaoundé Teaching Hospital)

Table 1. Characteristics of neonates born from HIV positive mothers, University of Yaoundé Teaching Hospital, Cameroon (December2001- November 2007).

\begin{tabular}{lll}
\hline & Mean $\left(\mathrm{SD}^{1}\right)$ & Median $\left(\mathrm{IQR}^{2}\right)$ \\
\hline Weight, g & $3025.5(599)$ & $3,093(2,752-3,388)$ \\
Length, cm & $48.9(3.2)$ & $50(48-51)$ \\
Head circumference, cm & $33.6(2.4)$ & $34(33-35)$ \\
Weight to length ratio & $2.56(0.36)$ & $2.53(2.34-2.74)$ \\
Gestational ages, weeks & $38.7(2.5)$ & $39(38-40)$ \\
Age (mothers), years & $27.9(4.8)$ & $27(25-31)$ \\
\hline
\end{tabular}

${ }^{1}$ SD: standard deviation. ${ }^{2} \mathrm{IQR}$ : interquartile range.

included and analysed in the study (Table 1). Ten neonates were twins (3.8\%) and 128 (48.5\%) were female. A total of 39 (14.8\%) had a LBW, 24 (9.1\%) had an IUGR and $36(13.6 \%)$ were premature. The mean birth weight, the mean birth length and the mean head circumference were $3026 \mathrm{~g}$ (SD: $\pm 599 \mathrm{~g}), 48.9 \mathrm{~cm}$ (SD: $\pm 3.2 \mathrm{~cm})$ and $33.6 \mathrm{~cm}$ (SD: $\pm 2.4 \mathrm{~cm}$ ), respectively; the median weight-to-length ratio was 2.53 (interquartile range [IQR]: 2.34 - 2.74); 26 (9.8\%) neonates had a weight-tolength ratio below the $10^{\text {th }}$ percentile (Table 1). A total of 259 HIV-positive mothers were included and analysed in the study (Table 2). The median age was 27 years (IQR: 25-31 years) at the time of delivery. A total of 149 (58\%) were married, and 148 (57\%) were jobless. Only $10(4 \%)$ HIV-positive mothers were illiterates. Most
Table 2. Characteristic of HIV-positive mothers, University of Yaoundé Teaching Hospital, Cameroon (December2001November 2007).

\begin{tabular}{cc}
\hline HIV classification stage 2 or 3, n (\%) & 28/259 (10.8) \\
\hline Syphilis, n (\%) & $8 / 259(3.1)$ \\
Anaemia, n (\%) & $34 / 259(13.1)$ \\
Single, n (\%) & $149 / 259(57.5)$ \\
Jobless, n (\%) & $148 / 259$ (57.1) \\
Education & \\
Illiterate, n (\%) & $10 / 259(3.9)$ \\
Primary school level, n (\%) & $67 / 259(25.9)$ \\
Secondary school level, n (\%) & $128 / 259(49.4)$ \\
University level, n (\%) & $54 / 259(20.8)$ \\
\hline
\end{tabular}

mothers ended school at secondary school level $[\mathrm{n}=128$ (49\%)], followed by 67 (26\%) who ended school at a primary school level, and 54 (21\%) who had a university degree.

Prenatal Care: A total of 96 (37\%) mothers were first para, 82 (32\%) second, 35 (13\%) third, and $46(18 \%)$ fourth para or more. Most (202; 78\%) attended 4 prenatal care visits or more, 30 (12\%) attended 3 visits, 22 (8\%) attended 2 visits, and 4 (2\%) attended only one visit. HIV was diagnosed among 89 (34\%) mothers before pregnancy, among 74 (29\%) mothers in the first trimester, among 63 (24\%) in the second trimester and among 33 (13\%) in the third trimester of pregnancy. Most HIVpositive mothers were at WHO-stage 1 at delivery [231 (89\%)], 23 (9\%) were at stage 2 and $5(2 \%)$ at stage 3. Only 13 (5\%) mothers were offered antiretroviral therapy (ART) during pregnancy. HIV laboratory parameters such as viral load, CD4 cell counts, and CD8 cell counts were obtained only for 6 (2\%) HIV-positive women. Anaemia prophylaxis was observed by the majority of the mothers 243 (94\%). There was significantly more anaemia ( $\mathrm{p}=0.003$ ) among mothers without taking a combination of folic acid and iron. Malaria prophylaxis was observed by 211 (81\%) mothers. Sixteen (6\%) were diagnosed with malaria in the third trimester or at delivery. There was no significant association between compliance with malaria prophylaxis and clinical disease $(\mathrm{p}=$ 0.20). Eight mothers (3\%) were diagnosed with syphilis and received benzathine penicillin treatment during pregnancy. No neonate was found with congenital syphilis.

Risk factor Analysis: Multivariate analysis showed that twins were significantly more at risk for LBW (OR [95\% CI]: 11.5 [3.1 - 43.5]; $\mathrm{p}<0.001)$, or being preterm (13.5 
[3.5-52.5]; $\mathrm{p}<0.001)$ than singletons. HIV-classification $>1$ was significantly associated with IUGR (OR [95\% CI): 3.15 [1.4-7.4]; $\mathrm{p}=0.01)$ and LBW (2.20 [1.1 - 4.6]; $\mathrm{p}=0.03)$ but not with prematurity $(\mathrm{p}=0.82)$. Risk factors for prematurity are summarized in Table 3 . Less than 4 prenatal care visits were associated with prematurity (OR [95\% CI]: 2.7 [1.21 - 6.05]; $\mathrm{p}=0.015$ ). Risk factors for IUGR are summarized in Table 4. Significant associations with IUGR were detected for lower gestational age $(\mathrm{p}<0.001)$ and when the clinical stage of the HIV-positive mother was higher than WHO-classification 1 at delivery. Although not significant, a trend for malaria disease in pregnancy (OR [95\% CI]: 3.82 [0.93 - 15.7]; p $=0.06$ ) was detected for IUGR.
WHO-classification 3 (OR [95\% CI]: 11.0 [1.68 72.0]; $\mathrm{p}=0.01$ ), malaria disease in pregnancy (OR [95\% $\mathrm{CI}]: 3.90$ [1.05 - 14.5]; $\mathrm{p}=0.04)$ and syphilis in pregnancy (OR [95\% CI]: 6.87 [1.38 - 34.1]; p = 0.02) were significantly associated with a weight-to-length ratio below the $10^{\text {th }}$ percentile.

Higher education was significantly associated with attending 4 or more prenatal care visits (OR [CI95\%]: 2.4 [1.6 - 3.4]; p < 0.001); similarly, women with higher education were more likely to respect anaemia prophylaxis (OR [CI95\%]: 3.0 [1.5 - 5.8]; p = 0.002) and antimalaria prophylaxis (OR [CI95\%]: 2.1 [1.4 - 3.3]; p < 0.001). Furthermore, higher education was associated with early HIV-diagnosis $(\mathrm{p}=0.003)$.

Table 3. Risk factor analysis for prematurity (gestational age $<37$ weeks) among neonates born from HIV positive mothers, University of Yaoundé Teaching Hospital, Cameroon (December2001 - November 2007).

\begin{tabular}{lllllll}
\hline & \multicolumn{3}{l}{ Univariate model } & \multicolumn{3}{c}{ Multivariate model } \\
\cline { 2 - 6 } & OR & P & $95 \%$ CI & OR & P & $95 \%$ CI \\
\hline Prenatal care visits $<4$ & 0.48 & 0.06 & $0.22-1.04$ & 2.71 & 0.015 & $1.21-6.05$ \\
Anaemia & 1.43 & 0.47 & $0.55-3.74$ & - & - & - \\
Malaria & 0.90 & 0.89 & $0.20-4.13$ & - & - & - \\
HIV stage $>1$ & 1.11 & 0.82 & $0.46-2.66$ & - & - & - \\
Syphilis & 0.90 & 0.92 & $0.11-7.56$ & - & - & $3.53-54.41$ \\
Twins & 11.2 & $<0.001$ & $2.97-42.8$ & 13.8 & $<0.001$ & - \\
Sex & 0.82 & 0.58 & $0.41-1.66$ & - & - & $0.89-1.48$ \\
Mother age at delivery & 1.19 & 0.15 & $0.94-1.52$ & 1.15 & 0.29 & - \\
Previous pregnancy & 1.13 & 0.44 & $0.83-1.55$ & - & - & - \\
Marital status & 0.70 & 0.33 & $0.34-1.45$ & - & - & - \\
Higher education & 1.03 & 0.91 & $0.65-1.62$ & - & - & - \\
Income & 1.17 & 0.67 & $0.58-2.36$ & - & - & - \\
\hline
\end{tabular}

Table 4. Risk factor analysis for IUGR (Weight $<10^{\text {th }}$ percentile) among neonates born from HIV positive mothers, University of Yaoundé Teaching Hospital, Cameroon (December2001 - November 2007).

\begin{tabular}{|c|c|c|c|c|c|c|}
\hline & \multicolumn{3}{|c|}{ Univariate model } & \multicolumn{3}{|c|}{ Multivariate model } \\
\hline & OR & $\mathbf{P}$ & $95 \% \mathrm{CI}$ & OR & $\mathrm{P}$ & $95 \% \mathrm{CI}$ \\
\hline Gestational age & 0.76 & $<0.001$ & $0.66-0.872$ & 0.75 & $<0.001$ & $0.64-0.87$ \\
\hline Prenatal care visits $<4$ & 0.71 & 0.20 & $0.42-1.19$ & 0.78 & 0.45 & $0.41-1.48$ \\
\hline Anaemia & 0.96 & 0.95 & $0.27-3.41$ & - & - & - \\
\hline Malaria & 2.49 & 0.17 & $0.65-9.45$ & 3.8 & 0.063 & $0.93-15.7$ \\
\hline HIV stage $>1$ & 3.16 & 0.003 & $1.49-6.71$ & 3.15 & 0.008 & $1.35-7.36$ \\
\hline Syphilis & 1.44 & 0.73 & $0.17-12.28$ & - & - & - \\
\hline Twins & 2.62 & 0.24 & $0.52-13.13$ & - & - & - \\
\hline Sex & 1.12 & 0.78 & $0.48-2.60$ & - & - & - \\
\hline Previous pregnancy & 0.85 & 0.45 & $0.57-1.28$ & - & - & - \\
\hline Marital status & 1.59 & 0.27 & $0.68-3.71$ & - & - & - \\
\hline Higher education & 0.73 & 0.26 & $0.43-1.26$ & - & - & - \\
\hline Income & 1.30 & 0.53 & $0.56-3.02$ & - & - & - \\
\hline
\end{tabular}




\section{Discussion}

This is the first and largest cohort study on neonates born to HIV-positive mothers in Central Africa. Three modifiable risk factors for LBW, IUGR or prematurity were detected: 1) poor prenatal care; 2) maternal HIV clinical stage of WHO-classification $>1$ at delivery; 3 ) and syphilis and malaria disease in pregnancy, respectively. While an advanced clinical stage of HIV infection was associated with prematurity as well as IUGR, poor prenatal care was associated with prematurity and syphilis or malaria was associated with IUGR.

Poor prenatal care in the absence of maternal illness has been shown to be associated with adverse outcome such as LBW in Pakistan and Madagascar [24-26]. A study which was done in the US did not show a difference between two groups of different numbers of prenatal care visits [27]. However, the mean number of visits was high (9 visits vs. 14 visits) compared to our population as well as to other populations in developing countries. Prenatal care visits among HIV-positive women must be considered much more important. The finding that prematurity is associated with less prenatal care visits among a population without antiretroviral treatment as ours suggests that non-HIV-specific care is fundamental in the prevention of prematurity. Prenatal care includes physical examination, control of fetal growth and development, giving advice for a healthy diet, and motivating mothers to respect prophylaxis for malaria, anaemia and neural tube defects. Women attending prenatal care visits are more likely to respect such recommendations as we have seen in our population where the association between the number of consultations and compliance with anaemia prophylaxis was significant ( $\mathrm{p}<0.001)$.

Women with higher education were significantly more likely to attend prenatal care visits and to observe prophylaxis recommendations for anaemia, neural tube defects and malaria in our study. However, education level as well as other maternal socio-economic factors such as being married, having a job, or having regular income was not found to be significantly associated with LBW, IUGR or prematurity. This is most likely due to the study size. Maternal illiteracy has been shown to be a risk for adverse outcome in pregnancy $[6,7,12,16]$. Similarly, even if a direct association between maternal education and LBW, IUGR or prematurity could not have been shown in our study, women with higher education attended prenatal care more often and were more likely to respect advice for prophylaxis. Higher education was found more often among unmarried women. Thus, unmarried parenting women in our study were more likely to seek prenatal care. The finding that most HIV-positive women in our study were single represents an overall trend in Cameroon. The fact that single mothers in our study were more likely to seek help from hospital institutions can be interpreted as a positive finding. Once ART is made widely available for the treatment of pregnant women, regular prenatal care visits are essential. Thus it is good to know that single mothers who represent the majority of pregnant HIV-positive women can be motivated for regular prenatal controls. On the other hand, in industrialized countries it has been shown that prenatal stress is associated with prematurity and LBW [28]. Single parenting women usually face considerable pressure while working and caring for a child already when being pregnant and being HIV-positive makes this even worse. Further studies would have to reveal what "stress" means in the setting of a developing country such as Cameroon and whether the association would be similar.

The finding that illiterate women are less likely to attend prenatal care visits is troubling. Such women are more often married and although they have a regular income and their nutritional status may be better they depend largely on their husbands while their illiteracy is a handicap to find a job. This prevents them from obtaining appropriate information about precaution measures in pregnancy. As a consequence, they have a low compliance with prophylaxis measures and worse, HIV-infection often is diagnosed not before the third trimester or at delivery $(\mathrm{p}<0.001)$. In the light of efficient HIV-prophylaxis by ART during pregnancy which are now - available this finding is worrying. Much effort will have to be invested in reaching and motivating married women to get tested for HIV before or early in pregnancy and to attend regular prenatal care visits.

Our risks for adverse outcomes such as LBW, IUGR and prematurity were similar to a study among 1500 women for maternal risk factors [29]. A total of $11.7 \%$ were HIV-positive and $9.9 \%$ had malaria $(61 \%$ in our study). Rates for prematurity were similar but our study showed a higher proportion of LBW (15\% vs. 8\%). Similar to our study there was a significant association between IUGR and maternal malaria in pregnancy. Our numbers are in line also with others studies in developing countries $[9-11,16,30]$. It is somewhat surprising that our rates of adverse outcome were not much higher considering the fact that all women we included in our study were HIV-positive. The most likely explanation for this is the fact that most HIV-positive women 236 (91\%) in our study had HIV-disease of WHO-classification 1. Consequently, HIV-disease as measured by the WHOclassification in our study was independently associated with IUGR. HIV-status alone does not predict adverse outcome but severity of HIV-disease and AIDS does. Thus, a population of HIV-positive women should not be considered to be homogeneous. 
The main limitation of our study is the study size although we describe the largest cohort of neonates born to HIV-positive women. Given the high proportion of women representing HIV-classification 1 our cohort almost matches a normal population and associations between socio-economic factors and stress most likely are too small to detect in a cohort of 259 mothers and their 264 children. Larger studies must address this issue. A further shortcoming is that only half the children born to HIV-positive mothers were included in the study because the lack of information would have been unmanageable otherwise. Especially information of women excluded because they had no prenatal care visit at UYT would be of interest as many of them may not have attended any prenatal care visit at all.

In summary, this is the first and largest cohort study about neonates born to HIV-positive mothers in the Central Africa sub-region. The study showed that, in a relatively healthy HIV-population with most women of WHO-classification 1, prenatal care other than HIVcontrol significantly improves outcome in pregnancy. Single women with higher education were more likely to attend prenatal care visits and comply with prevention measures for anaemia and malaria. Furthermore, HIV was diagnosed earlier in this population. Women in family settings on the other side were less likely to seek assistance in the hospital for pregnancy and HIV was diagnosed rather late. In the future, more effort must be invested to address women in family settings in Cameroon in order to 1) early diagnose HIV; and 2) offer HIV-treatment in time and increase compliance with preventive strategies for adverse outcomes such as anaemia, malaria and neural tube defects of the child.

\section{Acknowledgements}

We thank Christine Ngo-Gweth, Brice Tagne-Kengny and Mr. Anaba as well as the team from the labour room of UYT for their valuable contribution to the study.

\section{REFERENCES}

[1] M.C. McCormick, "The contribution of low birth weight to infant mortality and childhood morbidity," New England Journal of Medicine, Vol. 312, No. 2, 1985, pp. 82-90. doi:10.1056/NEJM198501103120204

[2] F.W. Oechsli, "Ultrasound fetoscopy and intrauterine growth retardation: two misused fashionable ideas," Paediatr Perinat Epidemiol, Vol. 4, No. 2, 1990, pp. 8-12. doi:10.1111/j.1365-3016.1990.tb00612.x

[3] H.C. Chase, "Infant mortality and weight at birth: 1960 United States birth cohort," American Journal of Public Health Nations Health,Vol. 42, NO. 9, 1969, pp. 1618-1628. doi:10.2105/AJPH.59.9.1618

[4] R.B. Jensen, M. Chellakooty, S. Vielwerth, A. Vaag, T.
Larsen and G. Greisen, et al, "Intrauterine growth retardation and consequences for endocrine and cardiovascular diseases in adult life: does insulin-like growth factor-I play a role?” Hormone Research, Vol. 60, No. 3, 2003; pp. 136-148. doi:10.1159/000074515

[5] R.E. Grunau, M.F. Whitfield and C. Davis, "Pattern of learning disabilities in children with extremely low birth weight and broadly average intelligence," Archives of Pediatrics \& Adolescent Medicine, Vol. 156, No. 6, 2002, pp. 615-620.

[6] M.S. Kramer, "Determinants of low birth weight: methodo- logical assessment and meta-analysis,” Bull World Health Organ, Vol. 65, No. 5, 1987, pp. 663-737.

[7] P.B. Bloland, J.J. Wirima, R.W. Steketee, B. Chilima, A. Hightower and J.G. Breman, "Maternal HIV infection and infant mortality in Malawi: evidence for increased mortality due to placental malaria infection,” AIDS, Vol. 9, No. 7, pp. 721-726. doi:10.1097/00002030-199507000-00009

[8] C. Ticconi, M. Mapfumo, M. Dorrucci, N. Naha, E. Tarira and A. Pietropolli, et al, "Effect of maternal HIV and malaria infection on pregnancy and perinatal outcome in Zim- babwe,” Journal of Acquired Immune Deficiency Syndromes, Vol. 34, NO. 3, 2003, pp. 289-294. doi:10.1097/00126334-200311010-00005

[9] J.G. Ayisi, A.M. van Eijk, F.O. ter Kuile, M.S. Kolczak, J.A. Otieno and A.O. Misore, et al, "The effect of dual infection with HIV and malaria on pregnancy outcome in western Kenya,” AIDS, Vol. 17, No. 4, 2003, pp. 585-594.doi:10.1097/00002030-200303070-00014

[10] F.O. ter Kuile, M.E. Parise, F.H. Verhoeff, V. Udhayakumar, R.D. Newman and A.M. van Eijk, et al, "The burden of co-infection with human immunodeficiency virus type 1 and malaria in pregnant women in sub-saharan Africa," American Journal of Tropical Medicine and Hygiene, Vol. 72, No.2, 2004, pp. 41-54.

[11] J.J. Mosoko and A.N. Affana, "Prevalence du VIH et Facteurs Associes," Institut National de la Statistique Ministere de la Plani- fication, de la Programmation du Developpement et de l'Amenagement du Territoire Yaounde, Cameroun and ORC Macro Calverton, Maryland, 2005, pp. 293-303.

[12] P. Brocklehurst and R. French, "The association between ma- ternal HIV infection and perinatal outcome: a systematic review of the literature and meta-analysism," British Journal Obstetrics and Gynaecology, Vol. 105, No. 8, 1998, pp. 836-848.

[13] M.L. Dreyfuss, G.I. Msamanga, D. Spiegelman, D.J. Hunter, E.J. Urassa and E. Hertzmark, et al, "Determinants of low birth weight among HIV-infected pregnant women in Tanzania," American Journal of Clinical Nutrition, Vol. 74, No. 6, 2001, pp. 814-826.

[14] K. Castetbon, J. Ladner, V. Leroy, M. Chauliac, E. Karita and A. De Clercq, et al, "Low birthweight in infants born to African HIV-infected women: relationship with maternal body weight during pregnancy: Pregnancy and HIV Study Group (EGE),” Journal of Tropical Pediatrics, Vol. 
45, No. 3, 1999, pp. 152-7. doi:10.1093/tropej/45.3.152

[15] M. Bulterys, A. Chao, S. Munyemana, J.B. Kurawige, P. Naw- rocki and P. Habimana, et al, "Maternal human immunodefi- ciency virus 1 infection and intrauterine growth: a pro- spective cohort study in Butare, Rwanda," Pediatric Infectious Disease Journal, Vol. 13, No. 2, pp. 94-100. doi:10.1097/00006454-199402000-00003

[16] P. Lepage, P. Msellati, P. Van de Perre, D.G. Hitimana and F. Dabis, "Characteristics of newborns and HIV-1 infection in Rwanda," AIDS, Vol. 6, No. 8, 1992, pp. 882-883.

[17] P. Nair, L. Alger, S. Hines, S. Seiden, R. Hebel and J.P. Johnson, "Maternal and neonatal characteristics associated with HIV infection in infants of seropositive women," Journal of Acquired Immune Deficency Syndromes, Vol. 6, No. 3, 1993, pp. 298-302.

[18] T.E. Taha, G.A. Dallabetta, J.K. Canner, J.D. Chiphangwi, G. Liomba and D.R. Hoover, et al, "The effect of human im- munodeficiency virus infection on birthweight, and infant and child mortality in urban Malawi,” International journal of Epidemiology, Vol. 24, No. 5, 1995, pp. 1022-1029. doi:10.1093/ije/24.5.1022

[19] S. Weng, M. Bulterys, A. Chao, C.A. Stidley, A. Dushimimana and E. Mbarutso, et al, "Perinatal human immunodeficiency virus-1 transmission and intrauterine growth: a cohort study in Butare, Rwanda,” Pediatrics, Vol. 102, No. 2, 1998, p. 24. doi:10.1542/peds.102.2.e24

[20] J.B. Jackson, P. Musoke, T. Fleming, L.A. Guay, D. Bagenda and M. Allen, et al, "Intrapartum and neonatal single-dose nevi- rapine compared with zidovudine for prevention of mother-to-child transmission of HIV-1 in Kampala, Uganda: 18-month follow-up of the HIVNET 012 ran- domised trial,” Lancet, Vol. 362, No. 9387, 2003, pp. 859-868. doi:10.1016/S0140-6736(03)14341-3

[21] L.O. Lubchenco, C. Hansman and E. Boyd, "Intrauterine growth in length and head circumference as estimated from live births at gestational ages from 26 to 42 weeks,” Pediatrics, Vol. 37, No. 3, 1966, pp. 403-408.

[22] R. Teck, O. Ascurra, P. Gomani, M. Manzi, O. Pasulani and J. Kusamale, et al, "WHO clinical staging of HIV infection and disease, tuberculosis and eligibility for antiretroviral treatment: relationship to CD4 lymphocyte counts," International Journal of Tuberculosis and Lung
Disease, Vol. 9, No. 3, 2005, pp. 258-262.

[23] J. Schulte, K. Dominguez, T. Sukalac, B. Bohannon and M.G. Fowler, "Declines in low birth weight and preterm birth among infants who were born to HIV-infected women during an era of increased use of maternal antiretroviral drugs: Pediatric Spectrum of HIV Disease, 1989-2004," Pediatrics, Vol. 119, No. 4, 2007, pp. e900-6. doi:10.1542/peds.2006-1123

[24] R.C. Andriamady, J.M. Rasamoelisoa, H. Ravaonarivo and R.J. Ranjalahy, "Premature rupture of membranes seen at the Befelatanana maternity, Antananarivo University Hos- pital Center," Arch Inst Pasteur Madagascar Vol.65, No. 1-2, 1999, pp. 100-102.

[25] N. Tasnim, G. Mahmud and M.S. Arif, "Impact of reduced prenatal visit frequency on obstetric outcome in low-risk mothers,” Journal of College of Physicians Surgeons Pakistan, Vol. 15, No. 1, 2005, pp. 26-9.

[26] E.J. Kongnyuy, P.N. Nana, N. Fomulu, S.C. Wiysonge, L. Kouam and A.S. Doh, "Adverse perinatal outcomes of ado- lescent pregnancies in Cameroon,” Maternal and Child Health Journal, Vol. 12, No. 2, 2008, pp. 149-154. doi:10.1007/s10995-007-0235-y

[27] R.S. McDuffie Jr., A. Beck, K. Bischoff and J. Cross, "Orleans M. Effect of frequency of prenatal care visits on perinatal outcome among low-risk women. A randomized con- trolled trial," Journal of the American Medical Association, Vol. 275, No. 11, 1996, pp. 847-851. doi:10.1001/jama.275.11.847

[28] P.H. Rondo, R.F. Ferreira, F. Nogueira, M.C. Ribeiro, H. Lobert and R. Artes, "Maternal psychological stress and distress as predictors of low birth weight, prematurity and intrauter- ine growth retardation," European Journal of Clinical Nutrition, Vol. 57, No. 2, 2003, pp. 266-72. doi:10.1038/sj.ejcn.1601526

[29] D. Watson-Jones, H.A. Weiss, J.M. Changalucha, J. Todd, B. Gumodoka and J. Bulmer, et al, "Adverse birth outcomes in United Republic of Tanzania--impact and prevention of maternal risk factors," Bull World Health Organ, Vol. 85, No. 1, 2007, pp. 9-18. doi:10.2471/BLT.06.033258

[30] M.S. Kramer, "The epidemiology of adverse pregnancy outcomes: an overview," Journal of Nutrition, Vol. 133, No. 5, Suppl.2, 2003, pp. 1592S-6S. 